\title{
Article
}

\section{COVID-19 Modulates Inflammatory and Renal Markers That May Predict Hospital Outcomes among African American Males}

\author{
Wendy Fonseca ${ }^{1}{ }^{(D}$, Nobuhiro Asai ${ }^{1}$, Kazuma Yagi ${ }^{1}$, Carrie-Anne Malinczak ${ }^{1}$, Gina Savickas ${ }^{2}$, Christine \\ C. Johnson ${ }^{3}$, Shannon Murray ${ }^{2}$, Edward M. Zoratti ${ }^{4}$, Nicholas W. Lukacs ${ }^{1,5}$, Jia Li ${ }^{3,+}$ \\ and Charles F. Schuler IV $5,6, *,+$ (i)
}

check for updates

Citation: Fonseca, W.; Asai, N.; Yagi, K.; Malinczak, C.-A.; Savickas, G.; Johnson, C.C.; Murray, S.; Zoratti, E.M.; Lukacs, N.W.; Li, J.; et al. COVID-19 Modulates Inflammatory and Renal Markers That May Predict Hospital Outcomes among African American Males. Viruses 2021, 13, 2415. https://doi.org/10.3390/ v13122415

\section{Academic Editors:}

Luis Martinez-Sobrido and Fernando Almazan Toral

Received: 9 August 2021

Accepted: 24 November 2021

Published: 2 December 2021

Publisher's Note: MDPI stays neutral with regard to jurisdictional claims in published maps and institutional affiliations.

Copyright: (c) 2021 by the authors. Licensee MDPI, Basel, Switzerland. This article is an open access article distributed under the terms and conditions of the Creative Commons Attribution (CC BY) license (https:/ / creativecommons.org/licenses/by/ $4.0 /)$.
1 Department of Pathology, University of Michigan, Ann Arbor, MI 48109, USA; wfaguila@med.umich.edu (W.F.); nobua@med.umich.edu (N.A.); kyagi@med.umich.edu (K.Y.); carrieam@med.umich.edu (C.-A.M.); nlukacs@med.umich.edu (N.W.L.)

2 Translational and Clinical Research Center, Department of Internal Medicine, Henry Ford Hospital, Detroit, MI 48202, USA; gsavick1@hfhs.org (G.S.); smurray6@hfhs.org (S.M.)

3 Department of Public Health Sciences, Henry Ford Hospital, Detroit, MI 48202, USA; cjohnso1@hfhs.org (C.C.J.); jli4@hfhs.org (J.L.)

4 Division of Allergy and Immunology, Department of Internal Medicine, Henry Ford Hospital, Detroit, MI 48202, USA; ezoratt1@hfhs.org

5 Mary H. Weiser Food Allergy Center, University of Michigan, Ann Arbor, MI 48109, USA

6 Division of Allergy and Clinical Immunology, Department of Internal Medicine, University of Michigan, Ann Arbor, MI 48109, USA

* Correspondence: schulerc@med.umich.edu; Tel.: 734-232-2154; Fax: 734-647-6263

+ Both authors contributed equally.

Abstract: Background and Objectives: African Americans and males have elevated risks of infection, hospitalization, and death from SARS-CoV-2 in comparison with other populations. We report immune responses and renal injury markers in African American male patients hospitalized for COVID-19. Methods: This was a single-center, retrospective study of 56 COVID-19 infected hospitalized African American males 50+ years of age selected from among non-intensive care unit (ICU) and ICU status patients. Demographics, hospitalization-related variables, and medical history were collected from electronic medical records. Plasma samples collected close to admission ( $\leq 2$ days) were evaluated for cytokines and renal markers; results were compared to a control group $(n=31)$ and related to COVID-19 in-hospital mortality. Results: Among COVID-19 patients, eight (14.2\%) suffered in-hospital mortality; seven (23.3\%) in the ICU and one (3.8\%) among non-ICU patients. Interleukin (IL)-18 and IL-33 were elevated at admission in COVID-19 patients in comparison with controls. IL-6, IL-18, MCP-1/CCL2, MIP-1 $\alpha$ /CCL3, IL-33, GST, and osteopontin were upregulated at admission in ICU patients in comparison with controls. In addition to clinical factors, MCP-1 and GST may provide incremental value for risk prediction of COVID-19 in-hospital mortality. Conclusions: Qualitatively similar inflammatory responses were observed in comparison to other populations reported in the literature, suggesting non-immunologic factors may account for outcome differences. Further, we provide initial evidence for cytokine and renal toxicity markers as prognostic factors for COVID-19 in-hospital mortality among African American males.

Keywords: COVID-19; SARS-CoV-2; cytokines; renal toxicity

\section{Introduction}

Severe acute respiratory syndrome-coronavirus-2 (SARS-CoV-2) is the $\beta$-coronavirus responsible for the COVID-19 global pandemic, causing millions of infections and deaths (according to the WHO). COVID-19 causes varied clinical manifestations, from mild forms to pneumonia and acute respiratory distress syndrome (ARDS) [1-3]. Three COVID-19 severity phenotypes are described: "mild" (80\%) with minor symptoms not progressing to 
severe disease, "moderate" (15\%) requiring hospitalization, and "severe" (5\%) requiring critical care management and at risk of a fatal outcome [4,5].

The immune reaction to SARS-CoV-2 infection can lead to cytokine storm syndrome, with substantial mortality risk [6,7]. Inflammatory mediators, such as interleukin (IL) IL-2, IL-7, IL-10, IL-1, IL-6, IL-18, IL-33, tumor necrosis factor (TNF), monocyte chemoattractant protein-1 (MCP-1; also known as CCL2), macrophage inflammatory protein 1 alpha (MIP$1 \alpha$; also known as CCL3), CXC-chemokine ligand 10 (CXCL10), RANTES (also known as CCL5), C-reactive protein (CRP), ferritin, and D-dimer, are upregulated systemically in severe COVID-19 cases [6,7]. Anti-inflammatory treatments targeting IL-6 and IL-1, as well as hemoperfusion techniques, have shown evidence of utility in severe COVID19 , suggesting an exacerbated inflammatory response partly mediates severity [8-10]. SARS-CoV-2 can therefore have systemic effects [11,12]; for example, renal disease is also associated with in-hospital mortality in COVID-19 [12,13]. Renal failure for severe COVID19 may be multifactorial as the virus causes cytokine storm, endothelial dysfunction, hypercoagulability, and/or sepsis $[5,12,14]$.

The Centers for Disease Control and Prevention (CDC) has reported that sex as well as race and ethnicity are risk factors for COVID-19 rates and severity via factors including socioeconomic status, healthcare access, co-morbidities, and occupational and environmental exposure [15]. Males in particular have an elevated mortality risk [9]. In the United States (US), the pandemic has disproportionately affected minority communities, particularly African Americans [16]; reports suggest the African American population has up to $1.4 \times$ the rate of SARS-CoV-2 infection, $3.7 \times$ the rate of hospitalization, and $2.8 \times$ the rate of mortality versus white, non-Hispanic, or Latino persons (CDC). In the state of Michigan, USA, African Americans represent $14 \%$ of the state population but up to $37 \%$ of the positive COVID-19 cases and $42 \%$ of COVID-19 deaths $[15,17,18]$. The African American community's disproportionate disease severity has been attributed to race, socioeconomic, and health disparities [17,18]; however, African American mortality is no worse than other groups once admitted to the hospital [19-21].

In this work, we aimed to understand how SARS-CoV-2 infection and inflammation systemically affects older African American males by studying clinical characteristics, the systemic immune response, and renal toxicity markers in a single-center retrospective cohort study of hospitalized COVID-19 patients.

\section{Materials and Methods}

\subsection{Ethics Approval}

All human studies were performed following University of Michigan (approval HUM00180532) and Henry Ford Health System (approval 13297) institutional review board-approved protocols. All participants provided written informed consent prior to specimen collection and enrollment in the Henry Ford Health System COVID-19 biobank. The institutional review board at Henry Ford Health System approved the biospecimen collection protocol and banking. Subsequently, samples requested for this project and accompanying clinical information were evaluated in a de-identified manner.

\subsection{Study Design and Patients}

From the start of COVID-19 in March 2020 in Michigan through September 2020, over 11,000 patients were admitted with COVID-19 to Henry Ford Health System (HFHS) in Southeast Michigan. A cohort of over 2000 patients were enrolled in the Translational and Clinical Research Center Biobank within HFHS. For this single-center, retrospective cohort study, we selected African American male patients $\geq 50$ years old, admitted for the first time to HFHS for COVID-19 infection from March to September 2020. Participants were admitted to the general or intensive care units (ICU). Patients on the general floor or ICU who were not ventilated at baseline but progressed to need a ventilator or who were stepped up in care to the ICU during their stay were excluded. COVID-19 status was defined by clinical nucleic acid amplification testing of nasopharyngeal swabs positive for 
SARS-CoV-2. No asymptomatic patients were included. The control cohort consisted of a convenience sample of 31 non-COVID-19 patients (inpatient or Emergency Department) encountered during the same time period with negative clinical nucleic acid amplification testing of nasopharyngeal swabs for COVID-19 with the same demographic characteristics; patients with a respiratory illness were excluded from this group, as were those who were presenting with an infectious disease.

Baseline patient characteristics collected included age, sex, past medical history, and medications. COVID-19-related symptoms and admission respiratory status were collected. Clinical outcomes collected included admission duration, level of care, respiratory care needs including mechanical ventilation status, COVID-19-related medications, and inhospital mortality. The earliest banked blood sample after hospital admission was used for all analyses. Median time between admission and blood drawn was $<1$ day; interquartile range was $0-3$ days.

\subsection{Multi-Analyte Immunoassay and Enzyme-Linked Immunosorbent Assay (ELISA)}

Cytokine levels of human IL-6, IL-18, MCP-1, MIP-1 $\alpha$, RANTES, TNF- $\alpha$, IFN- $\beta$, IFN- $\alpha 2$, and IFN- $\gamma$ were analyzed using a commercially available multi-analyte immunoassay (Bio-Plex cytokine assay, Bio-Rad Laboratories, Hercules, CA, USA). For the detection of IL-33 and IL-1 $\beta$, the R\&D Duo set ELISA kit (R\&D Systems, Minneapolis, MN, USA) was used per the manufacturer's instructions. Renal toxicity markers were evaluated using a commercial equipment Bio-Plex Pro RBM Human Kidney Toxicity Assays (Bio-Rad, Hercules, CA, USA) to measure glutathione S-transferase (GST), osteopontin, trefoil factor 3 (TFF3), neutrophil gelatinase-associated lipocalin (NGAL), calbindin, clusterin, kidney injury molecule-1 (KIM-1), and beta-2 microglobulin (B2M), following the manufacturer's instructions.

\subsection{Statistical Analysis}

Patient characteristics were summarized using standard descriptive statistics: mean/ standard deviation for continuous variables and frequency for categorical variables. To compare between the groups, we used the nonparametric Wilcoxon rank sum test for continuous variables and the Fisher's exact test for categorical variables.

Cytokines, in which more than $50 \%$ of samples were below the detection limit, were not included in the analysis. Distributions of the cytokine values were assessed and log2 transformed to render parametric statistical analyses. To compare cytokine/renal toxicity marker levels between groups, we performed two-sample $t$-tests or ANOVA. A sensitivity analysis based on nonparametric tests (Wilcoxon rank sum test or Kruskal-Wallis tests) was also conducted.

Additionally, we estimated the association between cytokine/renal toxicity markers with COVID-19 outcomes using the Firth logistic regression. Due to the small sample size and events, the ordinary logistic regression that is based on maximum likelihood estimation will yield biased estimates of coefficients. A penalized version such as Firth logistic regression is generally recommended for small-sample studies. Odds ratios of outcomes with a fold increase in marker levels were estimated. ORs were estimated on the basis of unadjusted and adjusted models. To correct for multiple testing issues, we adjusted $p$-values using the Benjamini-Hochberg approach for all above analyses. Adjusted $p$-values (FDR: false discovery rate) less than 0.1 or 0.05 are considered as suggestive cutoffs.

We next explored the potential of cytokines and renal toxicity markers as a complement variable for COVID-19 outcome risk stratification. Missing values for cytokines due to detection limit were imputed using quantile regression imputation of left-censored data before the analysis. The elastic-net logistic regression (ENET) was used to construct classifiers for in-hospital mortality. The tuning parameters of ENET were determined on the basis of the internal fivefold cross validation (CV). The tuning parameter limits the size of the coefficients and can result in models with small number of variables. The results were compared for a model based on patients' demographics and comorbidity, as well 
as a model based on patients' demographics, comorbidity, cytokines, and renal toxicity markers. The prediction metrics for comparison included receiver operating curves (ROC) and precision-recall curves (PRC). The whole process was evaluated by a repeated fivefold $\mathrm{CV}$. The goal is to test the model's ability to predict new data, to flag problems such as overfitting and selection bias, and to provide an insight on how the model will generalize to an independent external data set.

\section{Results}

\subsection{Patient Cohort}

A total of 56 COVID-19-affected and 31 non-COVID-19-affected African American male patients were analyzed (Table 1). Of those with COVID-19, 30 cases were treated in the ICU and 26 were hospitalized without requiring ICU care. ICU status patients had higher admission qSOFA scores. A total of $48 \%$ of COVID-19 patients were between the ages of 61 and 70. A total of 57\% of COVID-19 patients had a history of smoking, and these patients were less likely to have circulatory/vascular disease or a cancer history. More substance abuse and smoking were seen in non-COVID-19 patients. Four patients were on IL-6 cytokine inhibitors; these were excluded for the analysis of IL-6.

Table 1. Study participant characteristics by COVID-19 status.

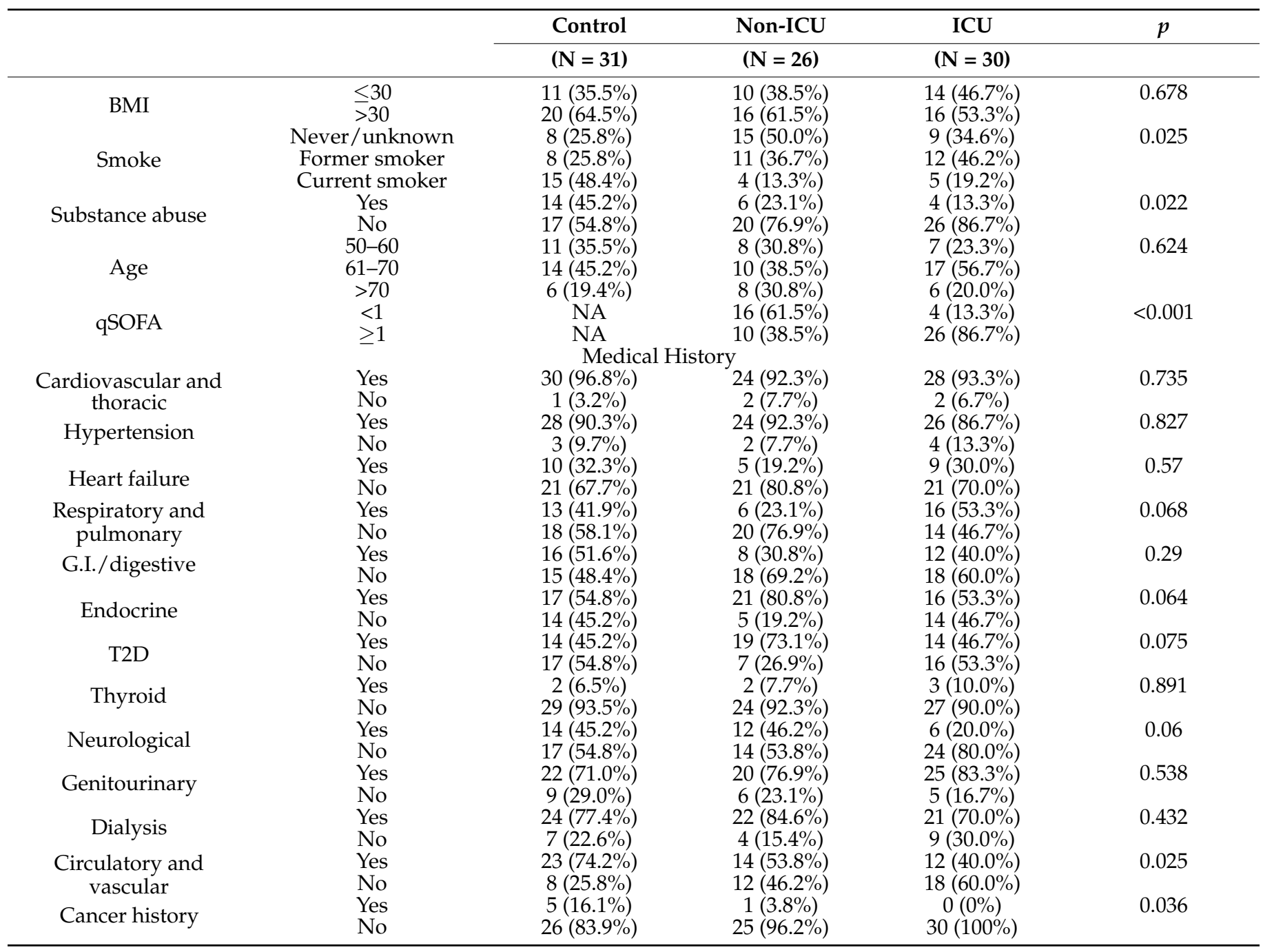

Patient characteristics and comorbidities were compared for in-hospital mortality among COVID-19 patients (Table 2). When comparing patient characteristics with in- 
hospital mortality, we found that higher qSOFA was associated with in-hospital mortality. More patients were on dialysis in the in-hospital mortality group (Table 2).

Table 2. COVID-19 patient characteristics by in-hospital mortality.

\begin{tabular}{|c|c|c|c|c|}
\hline & \multicolumn{4}{|c|}{ Hospital Death } \\
\hline & & Yes & No & \\
\hline \multirow{3}{*}{ BMI } & & $(\mathrm{N}=8)$ & $(\mathrm{N}=48)$ & \\
\hline & $\leq 30$ & $5(63 \%)$ & $27(56 \%)$ & $>0.999$ \\
\hline & $>30$ & $3(38 \%)$ & $21(44 \%)$ & \\
\hline \multirow{3}{*}{ Smoke } & Never/unknown & $4(50 \%)$ & $20(42 \%)$ & 0.54 \\
\hline & Former smoker & $4(50 \%)$ & $19(40 \%)$ & \\
\hline & Current smoker & $0(0 \%)$ & $9(19 \%)$ & \\
\hline \multirow{3}{*}{ Substance abuse } & Yes & $2(25 \%)$ & $8(17 \%)$ & 0.623 \\
\hline & No & $6(75 \%)$ & $40(83 \%)$ & \\
\hline & $50-60$ & $1(13 \%)$ & $14(29 \%)$ & 0.478 \\
\hline \multirow[t]{2}{*}{ Age } & $61-70$ & $4(50 \%)$ & $23(48 \%)$ & \\
\hline & $>70$ & $3(38 \%)$ & $11(23 \%)$ & \\
\hline \multirow{2}{*}{ qSOFA } & $<1$ & $0(0 \%)$ & $20(42 \%)$ & 0.041 \\
\hline & $\geq 1$ & $8(100 \%)$ & $28(58 \%)$ & \\
\hline \multirow{3}{*}{$\begin{array}{l}\text { Charleston comorbidity } \\
\text { index }\end{array}$} & $1-2$ & $0(0 \%)$ & $6(13 \%)$ & 0.588 \\
\hline & $3-4$ & $1(13 \%)$ & $10(21 \%)$ & \\
\hline & $>4$ & $7(88 \%)$ & $32(67 \%)$ & \\
\hline \multirow{3}{*}{ Cardiovascular and thoracic } & & Medical History & & \\
\hline & Yes & $8(100 \%)$ & $44(92 \%)$ & $>0.999$ \\
\hline & No & $0(0 \%)$ & $4(8 \%)$ & \\
\hline \multirow{2}{*}{ Hypertension } & Yes & $7(88 \%)$ & $43(90 \%)$ & $>0.999$ \\
\hline & No & $1(13 \%)$ & $5(10 \%)$ & \\
\hline \multirow{2}{*}{ Heart failure } & Yes & $0(0 \%)$ & $14(29 \%)$ & 0.18 \\
\hline & No & $8(100 \%)$ & $34(71 \%)$ & \\
\hline \multirow{2}{*}{ Respiratory and pulmonary } & Yes & $4(50 \%)$ & $18(38 \%)$ & 0.698 \\
\hline & No & $4(50 \%)$ & $30(63 \%)$ & \\
\hline \multirow{2}{*}{ G.I./digestive } & Yes & $2(25 \%)$ & $18(38 \%)$ & 0.697 \\
\hline & No & $6(75 \%)$ & $30(63 \%)$ & \\
\hline \multirow{2}{*}{ Endocrine } & Yes & $6(75 \%)$ & $31(65 \%)$ & 0.703 \\
\hline & No & $2(25 \%)$ & $17(35 \%)$ & \\
\hline \multirow{2}{*}{$\mathrm{T} 2 \mathrm{D}$} & Yes & $4(50 \%)$ & $29(60 \%)$ & 0.704 \\
\hline & No & $4(50 \%)$ & $19(40 \%)$ & \\
\hline \multirow{2}{*}{ Thyroid } & Yes & $2(25 \%)$ & $3(6 \%)$ & 0.144 \\
\hline & No & $6(75 \%)$ & $45(94 \%)$ & \\
\hline \multirow{2}{*}{ Neurological } & Yes & $1(13 \%)$ & $17(35 \%)$ & 0.414 \\
\hline & No & $7(88 \%)$ & $31(65 \%)$ & \\
\hline \multirow{2}{*}{ Genitourinary } & Yes & $7(88 \%)$ & $38(79 \%)$ & $>0.999$ \\
\hline & No & $1(13 \%)$ & $10(21 \%)$ & \\
\hline \multirow{2}{*}{ Dialysis } & Yes & $5(63 \%)$ & $8(17 \%)$ & 0.012 \\
\hline & No & $3(38 \%)$ & $40(83 \%)$ & \\
\hline \multirow{2}{*}{ Circulatory and vascular } & Yes & $3(38 \%)$ & $23(48 \%)$ & 0.712 \\
\hline & No & $5(63 \%)$ & $25(52 \%)$ & \\
\hline \multirow{3}{*}{ Cancer history } & Yes & $0(0 \%)$ & $1(2 \%)$ & $>0.999$ \\
\hline & No & $8(100 \%)$ & $47(98 \%)$ & \\
\hline & & Symptoms & & \\
\hline & Yes & $7(88 \%)$ & $30(65 \%)$ & 0.411 \\
\hline SOB & No & $1(13 \%)$ & $16(35 \%)$ & \\
\hline Couroh & Yes & $4(50 \%)$ & $26(57 \%)$ & $>0.999$ \\
\hline Cough & No & $4(50 \%)$ & $20(43 \%)$ & \\
\hline & Yes & $6(75 \%)$ & $22(48 \%)$ & 0.253 \\
\hline Fever/chills & No & $2(25 \%)$ & $24(52 \%)$ & \\
\hline
\end{tabular}


Table 2. Cont.

\begin{tabular}{|c|c|c|c|c|}
\hline & & \multicolumn{2}{|c|}{ Hospital Death } & \\
\hline & & Yes & No & \\
\hline \multirow{2}{*}{ Headache } & Yes & $0(0 \%)$ & $3(7 \%)$ & $>0.999$ \\
\hline & No & $8(100 \%)$ & $43(93 \%)$ & \\
\hline \multirow{2}{*}{ Nausea/diarrhea/vomiting } & Yes & $3(38 \%)$ & $15(33 \%)$ & $>0.999$ \\
\hline & No & $5(63 \%)$ & $31(67 \%)$ & \\
\hline \multirow{2}{*}{ Congestion } & Yes & $0(0 \%)$ & $2(4 \%)$ & $>0.999$ \\
\hline & No & $8(100 \%)$ & $44(96 \%)$ & \\
\hline Fatigue/body & Yes & $7(88 \%)$ & $19(42 \%)$ & 0.024 \\
\hline ache/weakness & No & $1(13 \%)$ & $26(58 \%)$ & \\
\hline \multirow{3}{*}{$\begin{array}{c}\text { Change in scent/smell/lack } \\
\text { of appetite }\end{array}$} & Yes & $0(0 \%)$ & $9(20 \%)$ & 0.324 \\
\hline & No & $8(100 \%)$ & $36(80 \%)$ & \\
\hline & \multicolumn{3}{|c|}{ Experimental/Relevant Medications } & \\
\hline \multirow{2}{*}{ Plaquenil/HCQ } & Yes & $8(100 \%)$ & $32(68 \%)$ & 0.091 \\
\hline & No & $0(0 \%)$ & $15(32 \%)$ & \\
\hline \multirow{2}{*}{ Remdesivir } & Yes & $0(0 \%)$ & $2(4 \%)$ & $>0.999$ \\
\hline & No & $8(100 \%)$ & $43(96 \%)$ & \\
\hline \multirow{2}{*}{ Steroids } & Yes & $8(100 \%)$ & $35(74 \%)$ & 0.178 \\
\hline & No & $0(0 \%)$ & $12(26 \%)$ & \\
\hline \multirow{2}{*}{ Azithromycin } & Yes & $1(13 \%)$ & $12(26 \%)$ & 0.664 \\
\hline & No & $7(88 \%)$ & $35(74 \%)$ & \\
\hline \multirow{2}{*}{ Cytokine Inhibitors } & Yes & $2(25 \%)$ & $3(6 \%)$ & 0.149 \\
\hline & No & $6(75 \%)$ & $44(94 \%)$ & \\
\hline \multirow{2}{*}{ NSAIDS } & Yes & $2(25 \%)$ & $14(30 \%)$ & $>0.999$ \\
\hline & No & $6(75 \%)$ & $33(70 \%)$ & \\
\hline
\end{tabular}

\subsection{Association of Cytokines and Renal Injury Markers with Demographics and} Baseline Comorbidities

We detected increases in IL-18, IL-6, and MIP-1 $\alpha$ in the COVID-19 ICU group versus controls and the non-ICU group (FDR $<0.05$; Figure 1 and Table S1). We also detected an increase in IL-33 and MCP-1 in the COVID-19 ICU group over controls (FDR < 0.05; Figure 1 and Table S1). Cytokines IL-18 and IL-33 were higher and IL-1 $\beta$ was lower in COVID-19 non-ICU patients versus controls (FDR $<0.1$ or $<0.05$ ). We also detected elevated GST and osteopontin in the ICU group versus controls (FDR $<0.05$, Figure 1). COVID-19 patients who had a genitourinary disorder had higher MCP-1 levels (FDR < 0.05; Figure S1). IL-1 $\beta$ levels were lower in COVID-19 patients with neurological disorders (FDR $<0.05$; Figure S1). There were no significant associations with age or BMI.

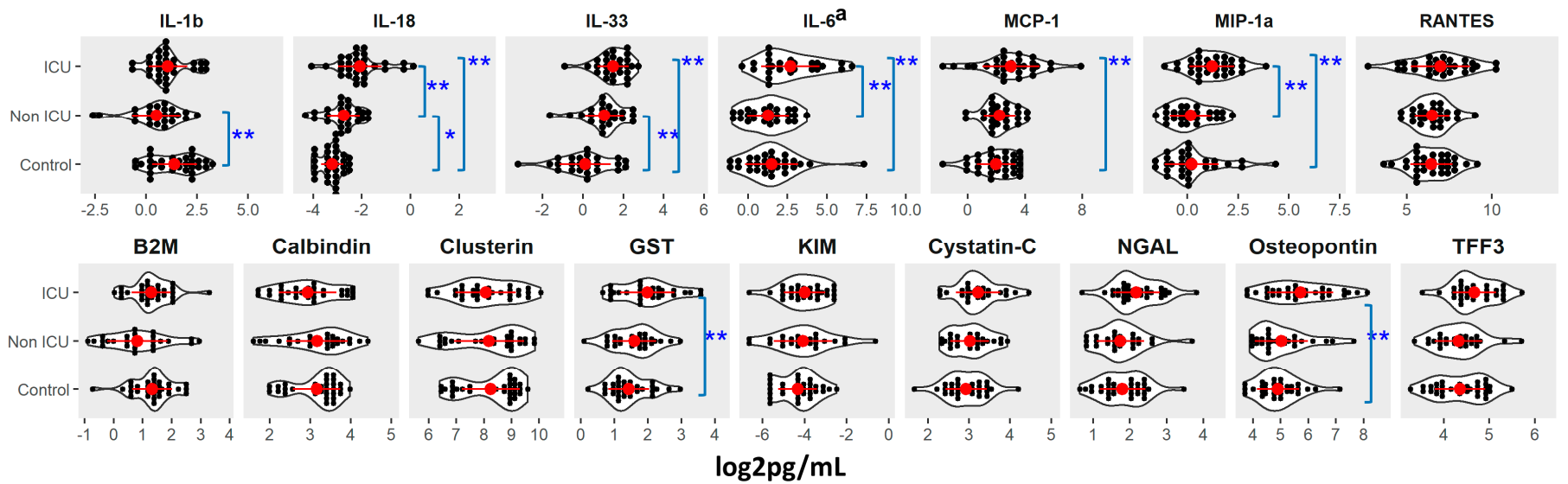

Figure 1. Cytokine and Renal toxicity marker levels by COVID-19 status at sample collection. *: adjusted $p$-value $($ FDR $) \leq 0.1{ }^{* *}$ : adjusted $p$-value $(F D R) \leq 0.05$. a: patients on cytokine inhibitor were excluded for analysis. Details were provided in Table S1. 
We detected elevated osteopontin and GST in samples from COVID-19 ICU patients versus controls (FDR < 0.05; Figure 1). COVID-19 patients who had dialysis or genitourinary disorder had higher levels of osteopontin, GST, and TFF3 (FDR $<0.1$ or $<0.05$; Figure S2).

\subsection{Association between Cytokines, Renal Injury Markers, and Risk of COVID-19 in-Hospital Death}

IL-33 was significantly higher in in-hospital non-survivors compared to survivors (FDR $<0.1$; Figure 2). GST, NGAL, and osteopontin were significantly elevated in nonsurvivors as well (FDR $<0.1$ or $<0.05$; Figure 2).

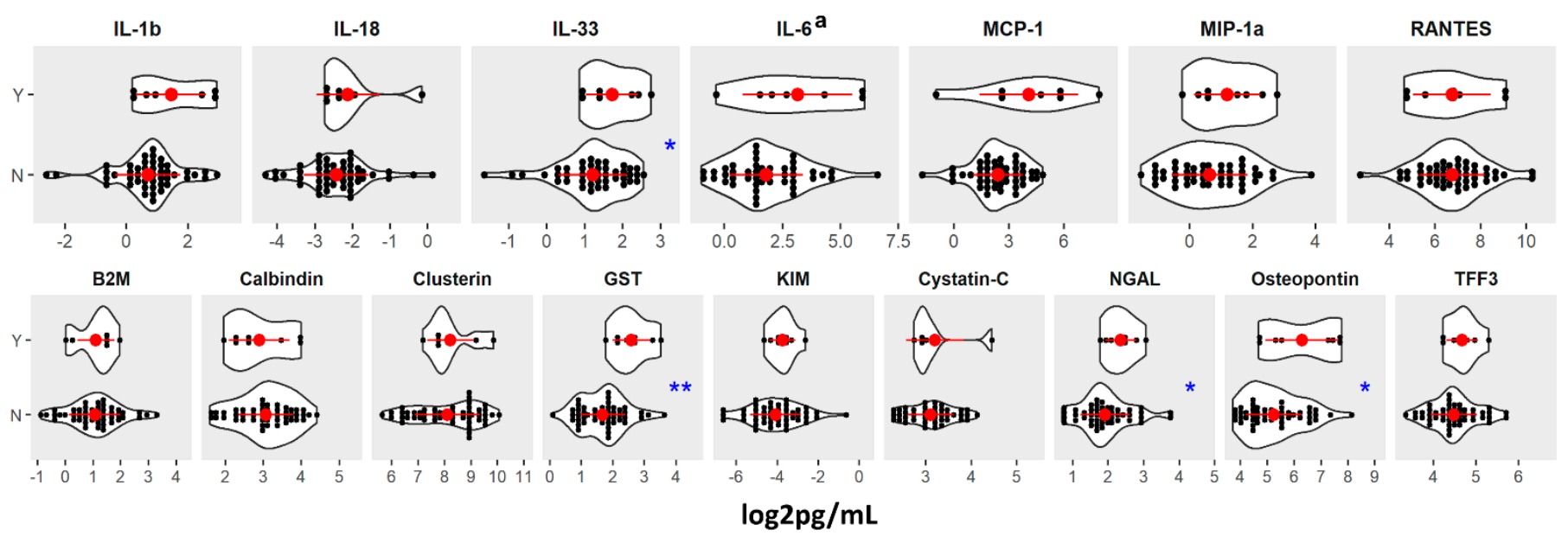

Figure 2. Cytokine and Renal toxicity marker levels by COVID-19 in-hospital death (Y vs. N). *: adjusted $p$-value $($ FDR $) \leq 0.1 ; *$ : adjusted $p$-value $(F D R) \leq 0.05$. a: patients on cytokine inhibitor were excluded for analysis.

We further estimated the odds ratio of in-hospital death with onefold increase in the marker levels from an unadjusted and adjusted model (see the Methods section). In the adjusted model, qSOFA score and dialysis were adjusted as potential confounders (time between blood draw and admission was also considered but did not change the conclusion). IL-6, MCP-1, and IL-33 were significantly associated with risk of in-hospital mortality; the results are marginal after adjustment for other variables (Figure 3). For renal injury markers, higher GST and osteopontin levels were associated with higher risk of in-hospital death, although again the results were marginal after adjustment for other variables (Figure 3).

We further considered using cytokine and renal injury markers to complement risk stratification. The ENAT model selected qSOFA score, history of heart failure, and dialysis as important clinical risk factors for in-hospital mortality. The area under the ROC (AUROC) was 0.69 (95\% CI: 0.47-0.91; Figure 4). In addition to clinical factors, MCP-1 and GST were independently predictive of patient in-hospital mortality; the AUROC of the secondary model was 0.74 (95\% CI: 0.56-0.92). It was significantly improved, and the $p$-value was found to be 0.038 , obtained by parametric bootstrap. Precision-recall curve was also higher when MCP-1 and GST were included compared to a clinical risk factor-only model (0.32 vs. $0.29 ; p$-value $=0.006)$. 

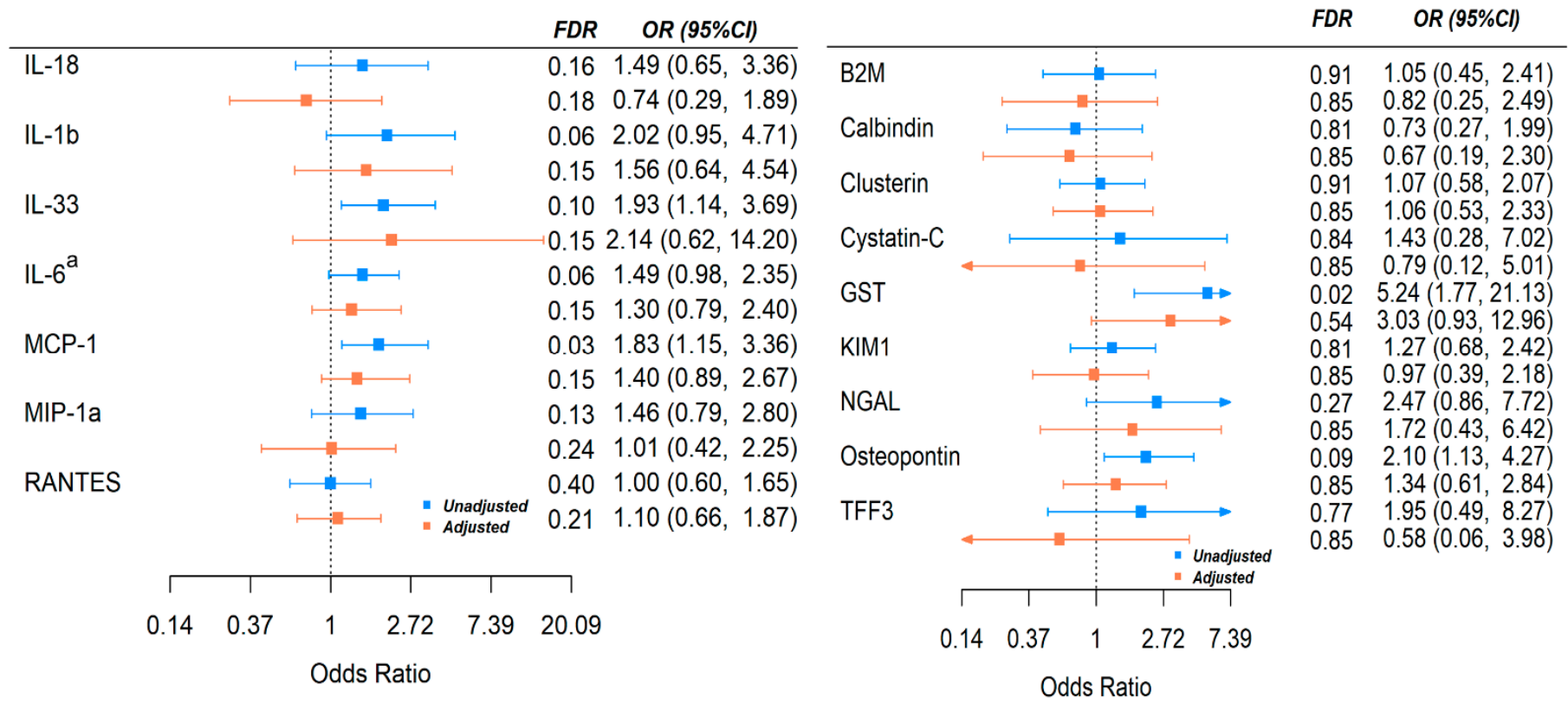

Figure 3. Association of Cytokines with risk of COVID-19 in-hospital mortality before and after adjustment for potential confounders. Adjusted model: dialysis, qSOFA. OR: odds ratio of outcome with 1-fold increase in cytokine levels. a: patients on cytokine inhibitor were excluded for analysis.

A. Mean ROC curve

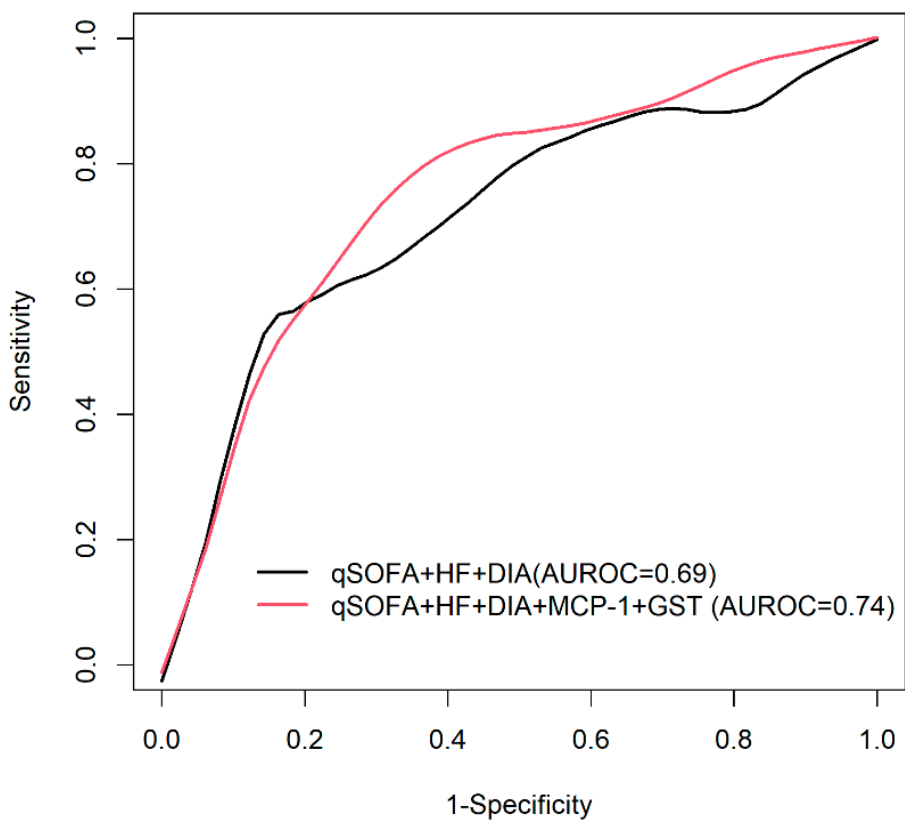

B. Mean Precision-Recall curve

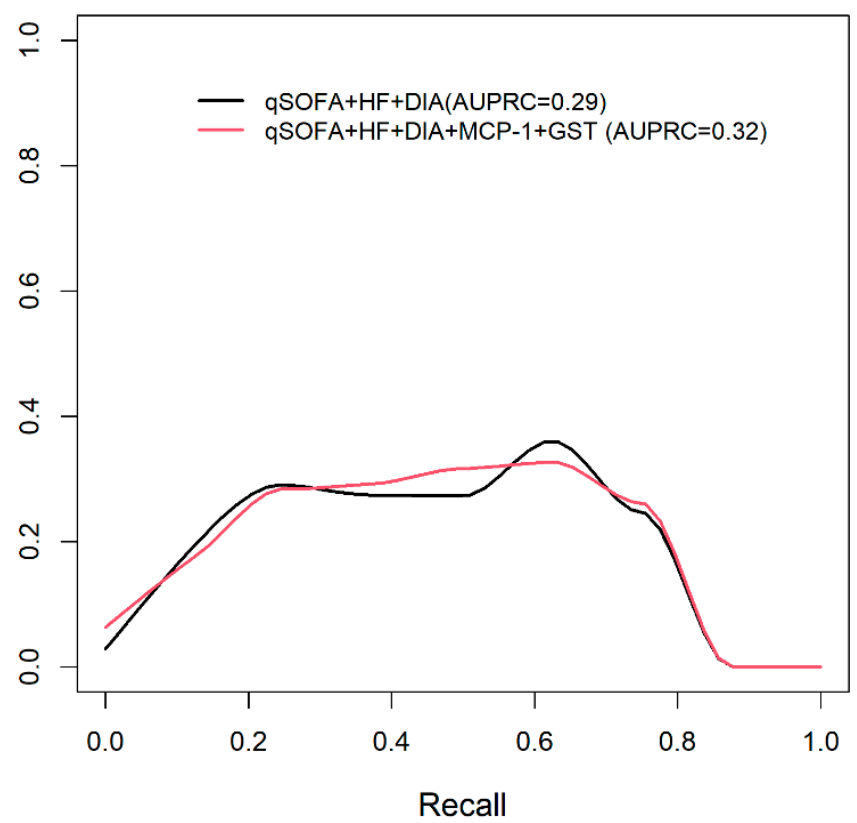

Figure 4. Comparison of prediction accuracy for clinical variable-based model versus cytokine and renal toxicity-based model for predicting in-hospital death in patients with COVID-19. qSOFA: qSOFA $\geq 1$ vs. <1; HF: heart failure; DIA: dialysis.

\section{Discussion}

SARS-CoV-2, the virus responsible for COVID-19, has caused a worldwide pandemic with millions of cases and deaths (WHO). An overwhelming systemic inflammatory response leading to multi-organ failure is associated with mortality and severe disease [6,7]. Males have an elevated mortality risk [9]. African Americans have been adversely impacted by this pandemic, with disproportionate hospitalizations and deaths, particularly early on in the pandemic timeline [22-25]. Evidence has emerged that African Americans do not 
have elevated in-hospital mortality over other groups once admitted to the hospital [18]. However, African American males have not been studied extensively to examine systemic inflammatory responses to COVID-19. We sought to define the systemic inflammatory response in this highest risk group and evaluate whether systemic inflammation and renal dysfunction markers described in previous reports could be applied to this population.

In this study, we observed key similarities in the systemic inflammatory response to prior reports. IL-6 and IL-18, among others, were elevated in ICU status COVID-19 patients versus non-ICU COVID-19 patients and hospitalized controls [9,26,27]. IL-33, MCP-1/CCL2, and MIP-1 $\alpha$ /CCL3 were also elevated in ICU status COVID-19 subjects over controls, consistent with other reports [28-31]. Interestingly, a clinical study in China showed that a similar set of cytokines and chemokines were associated with COVID-19 severity; levels of IL-6, IL-7, IL-10, IL-18, G-CSF, M-CSF, MCP-1, MCP-3, IP-10, MIG, and MIP-1 $\alpha$ were more upregulated in males than females [32]. A Norwegian study reported increased IL- 6 and MCP- 1 were associated with respiratory failure in COVID-19-positive patients [33]. Many of these cytokines, including IL-6, IL-18, IL-33, MCP-1, and MIP-1 $\alpha$, were elevated in the COVID-19 ICU and/or in-hospital mortality groups in our study [34].

Renal dysfunction is associated with in-hospital mortality in COVID-19 [12,13]. We evaluated whether markers of renal dysfunction were associated with ICU status and/or in-hospital mortality in the present study. GST and osteopontin were associated with COVID-19 ICU status; GST, NGAL, and osteopontin were elevated among the in-hospital mortality group. NGAL has been implicated in COVID-19-related in-hospital mortality previously [35]. A retrospective COVID-19 study from the University of Tokyo Hospital of 17 critically ill patients described upregulation in urinary NGAL levels in patients that developed acute kidney injury [36], suggesting the increased levels of renal toxicity markers in our study could be an indicator of future development of acute kidney injury and possibly related with systemic organ failure. In addition, GST is involved in cellular detoxification and neutralization of oxidative stress; it has been reported that two isoenzymes, GSTT1-1 and GSTM1-1, are genetically deleted in a high percentage of the human population, with significant ethnic differences [37]. A correlation of GSTT1 polymorphism and COVID-19 outcome has been suggested, although no clinical data have been presented to correlate these results [38]. These findings around NGAL, GST, and osteopontin in this population deserve further study, as they may correlate with renal dysfunction and/or in-hospital mortality in COVID-19 and might enhance detection of such dysfunction at early stages.

The present study has limitations. First, this was a single-center study, which limits the broad applicability without further research in larger groups. Second, we enrolled an all-male cohort; while this was done to study an under-evaluated, specific population disproportionately affected by the COVID-19 pandemic and remove variation in support of statistical power, this could limit broad applicability. The control group that was enrolled included hospitalized and emergency department patients and did not include patients with a respiratory illness; while this was done to provide a clear baseline value for cytokine and other measurements, this may limit the applicability of the study. Last, while we attempted to use plasma cytokines and renal toxicity markers as prognostic factors, the study was hindered by too small of a sample size to provide precise estimates of association and model predictability. Validation of these findings is needed in a larger-scale study.

In conclusion, this study restricted to African American male patients hospitalized with COVID-19 showed key qualitative similarities in the systemic inflammatory response to COVID-19 to other populations worldwide. In addition, the results suggest novel markers of renal dysfunction that may predict in-hospital mortality from COVID-19. While the African American population has suffered disproportionately from COVID-19, this study suggests that the pandemic's impact may not emanate from differences in the type of overall systemic inflammatory responses but perhaps from other factors that sort according to race and/or ethnicity, such as socioeconomics, occupational or other exposure patterns, or comorbidities. The potential predictive capacity of plasma cytokine and renal toxicity markers might be generalizable if confirmed in additional cohorts and could add 
to the existing predictive models being developed for COVID-19 mortality to help better understand mortality mechanisms in this disease.

Supplementary Materials: The following are available online at https://www.mdpi.com/article/ 10.3390/v13122415/s1, Figure S1: Cytokine levels by COVID-19 patient characteristics at baseline, Figure S2. Renal toxicity levels by COVID-19 patient characteristics at baseline, Table S1. Distribution of cytokine, renal toxicity marker levels by COVID status.

Author Contributions: Conceptualization, W.F., C.C.J., E.M.Z., N.W.L., J.L. and C.F.S.IV; data curation, W.F., N.A., K.Y., C.-A.M., G.S., S.M., J.L. and C.F.S.IV; formal analysis, W.F., N.A., K.Y., C.-A.M., G.S., J.L. and C.F.S.IV; funding acquisition, C.C.J. and C.F.S.IV; investigation, W.F., N.A., K.Y., C.-A.M., G.S., C.C.J., S.M., E.M.Z., N.W.L., J.L. and C.F.S.IV; methodology, W.F., N.A., K.Y., C.-A.M., G.S., C.C.J., S.M., E.M.Z., N.W.L., J.L. and C.F.S.IV; project administration, G.S., S.M., N.W.L., J.L. and C.F.S.IV; resources, W.F., C.-A.M., G.S., C.C.J., S.M., E.M.Z., N.W.L. and J.L.; software, N.A., K.Y., J.L. and C.F.S.IV; supervision, C.C.J., E.M.Z., N.W.L., J.L. and C.F.S.IV; validation, W.F. and J.L.; visualization, W.F., J.L. and C.F.S.IV; writing-original draft, W.F., J.L. and C.F.S.IV; writing—review and editing, W.F., N.A., K.Y., C.-A.M., C.C.J., S.M., E.M.Z., N.W.L., J.L. and C.F.S.IV All authors have read and agreed to the published version of the manuscript.

Funding: This work was supported by Henry Ford Health System and the Mary H. Weiser Food Allergy Center at the University of Michigan.

Institutional Review Board Statement: All human studies were performed following University of Michigan and Henry Ford Health System institutional review board-approved protocols.

Informed Consent Statement: Informed consent was obtained from all subjects involved in the study.

Data Availability Statement: The data presented in this study are available on request from the University of Michigan and Henry Ford Health System Data Offices. The data are not publicly available due to ethical reasons.

Acknowledgments: The authors acknowledge all the staff and participants who created the COVID19 Specimen Library within the Translational and Clinical Research Center Biobank at Henry Ford Hospital System; this resource was foundational to this work.

Conflicts of Interest: The authors declare no conflict of interest. The funders had no role in the design of the study; in the collection, analyses, or interpretation of data; in the writing of the manuscript; or in the decision to publish the results.

\section{References}

1. Jamilloux, Y.; Henry, T.; Belot, A.; Viel, S.; Fauter, M.; El Jammal, T.; Walzer, T.; François, B.; Sève, P. Should we stimulate or suppress immune responses in COVID-19? Cytokine and anti-cytokine interventions. Autoimmun. Rev. 2020, $19,102567$. [CrossRef]

2. Zhou, F.; Yu, T.; Du, R.; Fan, G.; Liu, Y.; Liu, Z.; Xiang, J.; Wang, Y.; Song, B.; Gu, X.; et al. Clinical course and risk factors for mortality of adult inpatients with COVID-19 in Wuhan, China: A retrospective cohort study. Lancet 2020, 395, 1054-1062. [CrossRef]

3. Grasselli, G.; Zangrillo, A.; Zanella, A.; Antonelli, M.; Cabrini, L.; Castelli, A.; Cereda, D.; Coluccello, A.; Foti, G.; Fumagalli, R.; et al. Baseline Characteristics and Outcomes of 1591 Patients Infected With SARS-CoV-2 Admitted to ICUs of the Lombardy Region, Italy. JAMA 2020, 323, 1574-1581. [CrossRef]

4. Siddiqi, H.K.; Mehra, M.R. COVID-19 illness in native and immunosuppressed states: A clinical-therapeutic staging proposal. J. Heart Lung Transpl. 2020, 39, 405-407. [CrossRef]

5. Fu, L.; Wang, B.; Yuan, T.; Chen, X.; Ao, Y.; Fitzpatrick, T.; Li, P.; Zhou, Y.; Lin, Y.F.; Duan, Q.; et al. Clinical characteristics of coronavirus disease 2019 (COVID-19) in China: A systematic review and meta-analysis. J. Infect. 2020, 80, 656-665. [CrossRef]

6. Henderson, L.A.; Canna, S.W.; Schulert, G.S.; Volpi, S.; Lee, P.Y.; Kernan, K.F.; Caricchio, R.; Mahmud, S.; Hazen, M.M.; Halyabar, O.; et al. On the Alert for Cytokine Storm: Immunopathology in COVID-19. Arthritis Rheumatol. 2020, 72, 1059-1063. [CrossRef] [PubMed]

7. Hojyo, S.; Uchida, M.; Tanaka, K.; Hasebe, R.; Tanaka, Y.; Murakami, M.; Hirano, T. How COVID-19 induces cytokine storm with high mortality. Inflamm Regen 2020, 40, 37. [CrossRef] [PubMed]

8. Stone, J.H.; Frigault, M.J.; Serling-Boyd, N.J.; Fernandes, A.D.; Harvey, L.; Foulkes, A.S.; Horick, N.K.; Healy, B.C.; Shah, R.; Bensaci, A.M.; et al. Efficacy of Tocilizumab in Patients Hospitalized with Covid-19. N. Engl. J. Med. 2020, 383, 2333-2344. [CrossRef] [PubMed] 
9. Huet, T.; Beaussier, H.; Voisin, O.; Jouveshomme, S.; Dauriat, G.; Lazareth, I.; Sacco, E.; Naccache, J.M.; Bezie, Y.; Laplanche, S.; et al. Anakinra for severe forms of COVID-19: A cohort study. Lancet Rheumatol. 2020, 2, e393-e400. [CrossRef]

10. Rampino, T.; Gregorini, M.; Perotti, L.; Ferrari, F.; Pattonieri, E.F.; Grignano, M.A.; Valente, M.; Garrone, A.; Islam, T.; Libetta, C.; et al. Hemoperfusion with CytoSorb as Adjuvant Therapy in Critically Ill Patients with SARS-CoV2 Pneumonia. Blood Purif. 2021, 50, 566-571. [CrossRef]

11. Raza, A.; Estepa, A.; Chan, V.; Jafar, M.S. Acute Renal Failure in Critically Ill COVID-19 Patients With a Focus on the Role of Renal Replacement Therapy: A Review of What We Know So Far. Cureus 2020, 12, e8429. [CrossRef] [PubMed]

12. Cheng, Y.; Luo, R.; Wang, K.; Zhang, M.; Wang, Z.; Dong, L.; Li, J.; Yao, Y.; Ge, S.; Xu, G. Kidney disease is associated with in-hospital death of patients with COVID-19. Kidney Int. 2020, 97, 829-838. [CrossRef]

13. Paek, J.H.; Kim, Y.; Park, W.Y.; Jin, K.; Hyun, M.; Lee, J.Y.; Kim, H.A.; Kwon, Y.S.; Park, J.S.; Han, S. Severe acute kidney injury in COVID-19 patients is associated with in-hospital mortality. PLOS ONE 2020, 15, e0243528. [CrossRef]

14. Ahmadian, E.; Hosseiniyan Khatibi, S.M.; Razi Soofiyani, S.; Abediazar, S.; Shoja, M.M.; Ardalan, M.; Zununi Vahed, S. COVID-19 and kidney injury: Pathophysiology and molecular mechanisms. Rev. Med. Virol. 2020, 31, e2176. [CrossRef] [PubMed]

15. Available online: https://doi.org/2019-ncov/covid-data/investigations-discovery/hospitalization-death-by-race-ethnicity.html (accessed on 1 August 2021).

16. Bwire, G.M. Coronavirus: Why Men are More Vulnerable to COVID-19 Than Women? SN Compr. Clin. Med. 2020, 2, 874-876. [CrossRef]

17. Quan, D.; Luna Wong, L.; Shallal, A.; Madan, R.; Hamdan, A.; Ahdi, H.; Daneshvar, A.; Mahajan, M.; Nasereldin, M.; Van Harn, M.; et al. Impact of Race and Socioeconomic Status on Outcomes in Patients Hospitalized with COVID-19. J. Gen. Intern Med. 2021, 36, 1302-1309. [CrossRef] [PubMed]

18. Price-Haywood, E.G.; Burton, J.; Fort, D.; Seoane, L. Hospitalization and Mortality among Black Patients and White Patients with Covid-19. N. Engl. J. Med. 2020, 382, 2534-2543. [CrossRef]

19. Suleyman, G.; Fadel, R.A.; Malette, K.M.; Hammond, C.; Abdulla, H.; Entz, A.; Demertzis, Z.; Hanna, Z.; Failla, A.; Dagher, C.; et al. Clinical Characteristics and Morbidity Associated With Coronavirus Disease 2019 in a Series of Patients in Metropolitan Detroit. JAMA Netw. Open 2020, 3, e2012270. [CrossRef]

20. Raharja, A.; Tamara, A.; Kok, L.T. Association between Ethnicity and Severe COVID-19 Disease: A Systematic Review and Meta-analysis. J. Racial Ethn. Health Disparities 2020, 8, 1563-1572. [CrossRef]

21. Pan, D.; Sze, S.; Minhas, J.S.; Bangash, M.N.; Pareek, N.; Divall, P.; Williams, C.M.; Oggioni, M.R.; Squire, I.B.; Nellums, L.B.; et al. The impact of ethnicity on clinical outcomes in COVID-19: A systematic review. EClinicalMedicine 2020, 23, 100404. [CrossRef] [PubMed]

22. Anaele, B.I.; Doran, C.; McIntire, R. Visualizing COVID-19 Mortality Rates and African-American Populations in the USA and Pennsylvania. J. Racial Ethn. Health Disparities 2021, 8, 1356-1363. [CrossRef]

23. Escobar, G.J.; Adams, A.S.; Liu, V.X.; Soltesz, L.; Chen, Y.I.; Parodi, S.M.; Ray, G.T.; Myers, L.C.; Ramaprasad, C.M.; Dlott, R.; et al. Racial Disparities in COVID-19 Testing and Outcomes: Retrospective Cohort Study in an Integrated Health System. Ann. Intern. Med. 2021, 174, 786-793. [CrossRef]

24. Wu, K.H.; Hornsby, W.E.; Klunder, B.; Krause, A.; Driscoll, A.; Kulka, J.; Bickett-Hickok, R.; Fellows, A.; Graham, S.; Kaleba, E.O.; et al. Exposure and risk factors for COVID-19 and the impact of staying home on Michigan residents. PLoS ONE 2021, 16, e0246447. [CrossRef] [PubMed]

25. Andrasfay, T.; Goldman, N. Reductions in 2020 US life expectancy due to COVID-19 and the disproportionate impact on the Black and Latino populations. Proc. Natl. Acad. Sci. USA 2021, 118, e2014746118. [CrossRef] [PubMed]

26. Laguna-Goya, R.; Utrero-Rico, A.; Talayero, P.; Lasa-Lazaro, M.; Ramirez-Fernandez, A.; Naranjo, L.; Segura-Tudela, A.; CabreraMarante, O.; Rodriguez de Frias, E.; Garcia-Garcia, R.; et al. IL-6-based mortality risk model for hospitalized patients with COVID-19. J. Allergy Clin. Immunol. 2020, 146, 799-807.e9. [CrossRef] [PubMed]

27. Satis, H.; Ozger, H.S.; Aysert Yildiz, P.; Hizel, K.; Gulbahar, O.; Erbas, G.; Aygencel, G.; Guzel Tunccan, O.; Ozturk, M.A.; Dizbay, M.; et al. Prognostic value of interleukin-18 and its association with other inflammatory markers and disease severity in COVID-19. Cytokine 2021, 137, 155302. [CrossRef]

28. Munitz, A.; Edry-Botzer, L.; Itan, M.; Tur-Kaspa, R.; Dicker, D.; Marcoviciu, D.; Goren, M.G.; Mor, M.; Lev, S.; Gottesman, T.; et al. Rapid seroconversion and persistent functional IgG antibodies in severe COVID-19 patients correlates with an IL-12p70 and IL-33 signature. Sci. Rep. 2021, 11, 3461. [CrossRef]

29. Burke, H.; Freeman, A.; Cellura, D.C.; Stuart, B.L.; Brendish, N.J.; Poole, S.; Borca, F.; Phan, H.T.T.; Sheard, N.; Williams, S.; et al. Inflammatory phenotyping predicts clinical outcome in COVID-19. Respir. Res. 2020, 21, 245. [CrossRef] [PubMed]

30. Hue, S.; Beldi-Ferchiou, A.; Bendib, I.; Surenaud, M.; Fourati, S.; Frapard, T.; Rivoal, S.; Razazi, K.; Carteaux, G.; Delfau-Larue, M.H.; et al. Uncontrolled Innate and Impaired Adaptive Immune Responses in Patients with COVID-19 Acute Respiratory Distress Syndrome. Am. J. Respir. Crit. Care Med. 2020, 202, 1509-1519. [CrossRef] [PubMed]

31. Li, S.; Jiang, L.; Li, X.; Lin, F.; Wang, Y.; Li, B.; Jiang, T.; An, W.; Liu, S.; Liu, H.; et al. Clinical and pathological investigation of patients with severe COVID-19. JCI Insight 2020, 5, 138070. [CrossRef] [PubMed]

32. Chi, Y.; Ge, Y.; Wu, B.; Zhang, W.; Wu, T.; Wen, T.; Liu, J.; Guo, X.; Huang, C.; Jiao, Y.; et al. Serum Cytokine and Chemokine Profile in Relation to the Severity of Coronavirus Disease 2019 in China. J. Infect. Dis. 2020, 222, 746-754. [CrossRef] [PubMed] 
33. Jontvedt Jorgensen, M.; Holter, J.C.; Christensen, E.E.; Schjalm, C.; Tonby, K.; Pischke, S.E.; Jenum, S.; Skeie, L.G.; Nur, S.; Lind, A.; et al. Increased interleukin- 6 and macrophage chemoattractant protein-1 are associated with respiratory failure in COVID-19. Sci. Rep. 2020, 10, 21697. [CrossRef]

34. Xu, Z.S.; Shu, T.; Kang, L.; Wu, D.; Zhou, X.; Liao, B.W.; Sun, X.L.; Zhou, X.; Wang, Y.Y. Temporal profiling of plasma cytokines, chemokines and growth factors from mild, severe and fatal COVID-19 patients. Signal Transduct. Target Ther. 2020, 5, 100. [CrossRef] [PubMed]

35. Abers, M.S.; Delmonte, O.M.; Ricotta, E.E.; Fintzi, J.; Fink, D.L.; de Jesus, A.A.A.; Zarember, K.A.; Alehashemi, S.; Oikonomou, V.; Desai, J.V.; et al. An immune-based biomarker signature is associated with mortality in COVID-19 patients. JCI Insight 2021, 6, e144455. [CrossRef] [PubMed]

36. Komaru, Y.; Doi, K.; Nangaku, M. Urinary Neutrophil Gelatinase-Associated Lipocalin in Critically Ill Patients With Coronavirus Disease 2019. Crit. Care Explor. 2020, 2, e0181. [CrossRef]

37. Bolt, H.M.; Thier, R. Relevance of the deletion polymorphisms of the glutathione S-transferases GSTT1 and GSTM1 in pharmacology and toxicology. Curr. Drug Metab. 2006, 7, 613-628. [CrossRef]

38. Saadat, M. An evidence for correlation between the glutathione S-transferase T1 (GSTT1) polymorphism and outcome of COVID-19. Clin. Chim. Acta 2020, 508, 213-216. [CrossRef] 